\title{
Dissociating the time courses of the cross-modal semantic priming effects elicited by naturalistic sounds and spoken words
}

\author{
Yi-Chuan Chen ${ }^{1} \cdot$ Charles Spence $^{1}$
}

Published online: 9 June 2017

(C) The Author(s) 2017. This article is an open access publication

\begin{abstract}
The present study compared the time courses of the cross-modal semantic priming effects elicited by naturalistic sounds and spoken words on visual picture processing. Following an auditory prime, a picture (or blank frame) was briefly presented and then immediately masked. The participants had to judge whether or not a picture had been presented. Naturalistic sounds consistently elicited a cross-modal semantic priming effect on visual sensitivity $\left(d^{\prime}\right)$ for pictures (higher $d^{\prime}$ in the congruent than in the incongruent condition) at the 350-ms rather than at the 1,000-ms stimulus onset asynchrony (SOA). Spoken words mainly elicited a cross-modal semantic priming effect at the 1,000-ms rather than at the 350-ms SOA, but this effect was modulated by the order of testing these two SOAs. It would therefore appear that visual picture processing can be rapidly primed by naturalistic sounds via cross-modal associations, and this effect is short lived. In contrast, spoken words prime visual picture processing over a wider range of prime-target intervals, though this effect was conditioned by the prior context.
\end{abstract}

Keywords Semantic $\cdot$ Multisensory $\cdot$ Audiovisual · Sensitivity $\cdot$ Priming

In daily life, hearing the sound of a dog barking is likely informative with regard to the identity of a creature that is glimpsed, albeit briefly (Chen \& Spence, 2010). Indeed, the presentation of either a naturalistic sound or spoken word

Yi-Chuan Chen

yi-chuan.chen@psy.ox.ac.uk

1 Crossmodal Research Laboratory, Department of Experimental Psychology, University of Oxford, 9 South Parks Road, Oxford OX1 3UD, UK enhances the sensitivity $\left(d^{\prime}\right)$ of visual object detection (Chen \& Spence, 2011; Lupyan \& Ward, 2013). Such results suggest that the meaning of the auditory cue facilitates visual processing and boosts the breakthrough of the visual stimulus into awareness cross-modally rather than simply giving rise to some sort of criterion change (note that the dog barking certainly induces a likely guess that the creature might be a dog as well).

The time courses of cross-modal semantic priming effects, however, appear to be different for naturalistic sounds and spoken words. Chen and Spence (2011) demonstrated that when leading the target picture by $346 \mathrm{~ms}$, only naturalistic sounds (rather than spoken words) elicited a semantic priming effect on visual picture sensitivity in a simple detection task (when judging whether a picture was present or not). These results were explained based on evidence suggesting that naturalistic sounds access their associated meaning faster than spoken words do (Chen \& Spence, 2013; Cummings et al., 2006; Saygin, Dick, \& Bates, 2005). The different processing times plausibly stem from the differing routes of semantic access for each type of auditory stimulus: Naturalistic sounds access semantic information directly, whereas spoken words have to access their meanings via lexical representations (Barsalou, Santos, Simmons, \& Wilson, 2008; Chen \& Spence, 2011; Glaser \& Glaser, 1989).

Lupyan and colleagues, on the other hand, demonstrated an advantage for spoken words over naturalistic sounds at longer SOAs (around 1,000 ms or more; Edmiston \& Lupyan, 2015; Lupyan \& Thompson-Schill, 2012 ${ }^{1}$ ). The participants in their studies had to verify whether the auditory cue (either a

\footnotetext{
${ }^{1}$ The interstimulus interval was 400,1000 , or $1500 \mathrm{~ms}$ in Lupyan and Thompson-Schill (2012). However, since they did not report the duration of the auditory cue, it is not possible to determine the corresponding SOAs. Nevertheless, the SOAs were certainly longer than the $346 \mathrm{~ms}$ in Chen and Spence (2011).
} 
naturalistic sound or a spoken word) and the subsequently presented picture matched or not. The results demonstrate that the participants' reaction times (RTs) were shorter for spoken words than for naturalistic sounds. Further evidence comes from an event-related potential (ERP) study: When a spoken word led a target picture by around $1,670 \mathrm{~ms}$, the P1 component associated with the picture (at 70-125 ms after onset) occurred earlier in the congruent than in the incongruent condition, but no such congruency effect was induced by naturalistic sounds (Boutonnet \& Lupyan, 2015). These results were explained in terms of spoken words being associated with semantic representations that are more abstract and categorical, thus providing a conceptual cue regarding a given object that is general rather than specific to a particular exemplar, as compared to naturalistic sounds (Edmiston \& Lupyan, 2015; Lupyan \& Thompson-Schill, 2012).

Given the different SOAs and methods, and given the different mechanisms proposed by previous research (Chen \& Spence, 2011; Edmiston \& Lupyan, 2015; Lupyan \& Thompson-Schill, 2012), we wanted to carefully examine the time courses of cross-modal semantic priming effects elicited by naturalistic sounds and spoken words. Two critical SOAs were chosen: The 350- ms SOA is close to the interval at which Chen and Spence (2011) demonstrated cross-modal semantic priming by naturalistic sounds (but not by spoken words) in a picture detection task. The 1,000-ms SOA (the interstimulus interval, ISI, was $500-650 \mathrm{~ms}$ ) corresponds to the ISI somewhere between 400 and $1,000 \mathrm{~ms}$ used by Lupyan and Thompson-Schill (2012), the conditions demonstrated a cross-modal semantic advantage for spoken words over naturalistic sounds. In Experiment 1, each participant was tested with only one of the SOAs, following the designs of Chen and Spence (2011) and Lupyan and Thompson-Schill (2012). In Experiment 2, participants were tested with both SOAs in a counterbalanced order. In this case, we further examined whether the time courses of cross-modal semantic priming effects are stable or modulated by prior context.

\section{Experiment 1}

\section{Method}

\section{Participants}

Forty volunteers (10 males, mean age 22.2 years) took part in this experiment in exchange for course credit or five pounds (UK sterling). The participants were native English speakers or bilinguals who had started to learn English by 5 years of age. All participants had normal or corrected-to-normal vision and normal hearing by self-report, and all were naïve as to the purpose of the study. Written informed consent was obtained prior to the start of the study. The study was approved by the Medical Sciences Inter Divisional Research Ethics Committee, University of Oxford (MSD-IDREC-C1-2014-143).

\section{Apparatus and stimuli}

The visual stimuli were presented on a 23-inch LED monitor controlled by a personal computer. The participants sat at a viewing distance of $58 \mathrm{~cm}$ from the monitor in a dimly lit chamber. Twenty-four outline-drawings (12 living and 12 nonliving things) taken from Snodgrass and Vanderwart (1980) and Bates et al. (2003), as well as their mirror images, were used as visual targets (see Appendix). Five pattern masks were created by overlapping 20 nonobject figures randomly selected from Magnié, Besson, Poncet, and Dolisi (2003). Each pattern covered an area of $5.9^{\circ} \times 5.9^{\circ}$, sufficient to completely occlude all of the target pictures.

The auditory stimuli ( 8 bit mono; $22500 \mathrm{~Hz}$ digitization) were presented over closed-ear headphones and ranged in loudness from 31 to $51 \mathrm{~dB}$ sound pressure level (SPL). The naturalistic sounds were those produced by each of the objects. The spoken words consisted of the most commonly agreed-upon name used to refer each picture (Bates et al. 2003; Snodgrass \& Vanderwart, 1980) and were produced by a female native English speaker. The naturalistic sound and the spoken word associated with the same picture were edited to have the same duration. The root mean square values of all of the auditory stimuli were equalized.

\section{Design}

Two within-participants factors, prime type (naturalistic sound or spoken word) and congruency (congruent or incongruent), and one between-participants factor, SOA (350 or 1,000 ms), were manipulated. Naturalistic sounds and spoken words were presented in separate blocks of trials. Congruent and incongruent trials were mixed within blocks: The auditory cue matched the picture in the congruent trials, but they belonged to different categories based on the fundamental living thing versus nonliving thing separation in the incongruent trials. Each SOA was tested with 20 participants.

All 24 pictures and their mirror images were presented once in each block - either one was presented in the congruent trial and the other in the incongruent trial (and they were swapped in another block). These trials were used to estimate the participant's hit rate in the congruent and incongruent conditions, respectively. An additional 48 picture-absent trials, consisting of an auditory cue and a blank frame, were presented to estimate the participant's false alarm (FA) rate. These 96 trials were presented in a completely randomized order. There were two blocks for both naturalistic sounds and spoken words, and the order of these two types of auditory stimuli was counterbalanced across participants. The participants were 
not given any information concerning the possible semantic congruency between the auditory cue and picture prior to taking part in the study.

\section{Procedure}

The participants initiated a block of trials by pressing the enter key on the keyboard in front of them. In each trial (see Fig. 1a), a blank frame was followed by either a frame with a picture or another blank for $17 \mathrm{~ms}$ (one frame at the screen refresh rate of $60 \mathrm{~Hz}$ ). The pattern mask was presented immediately thereafter; meanwhile, the participants had to decide whether they had seen a picture (irrespective of its identity) presented before the mask by pressing the space bar. The participants were informed that the task was not speeded, and they should only respond if they were sure that they had seen a picture (i.e., they should maintain a strict response criterion).
Prior to the start of the main experiment, all of the pictures and their matched names were presented on the monitor in a completely randomized order across participants. Each picture-name pair was presented for 1,500 ms and interleaved by a blank frame for $500 \mathrm{~ms}$. An easy practice session (eight trials with a picture duration of $33 \mathrm{~ms}$ ) and a harder practice session (16 trials with a picture duration of $17 \mathrm{~ms}$ ) were conducted prior to the main experiment. In the easy practice session, the accuracy had to reach $85 \%$, or it was repeated up to three times. The stimuli in the practice session were not used in the main experiment. The experiment lasted for approximately 30 minutes.

\section{Results}

For both naturalistic sounds and spoken words, the hit rate in the congruent and incongruent conditions was estimated on the basis of 48 trials ( 24 pictures $\times 2$ blocks), while the FA rate

(A) Procedure

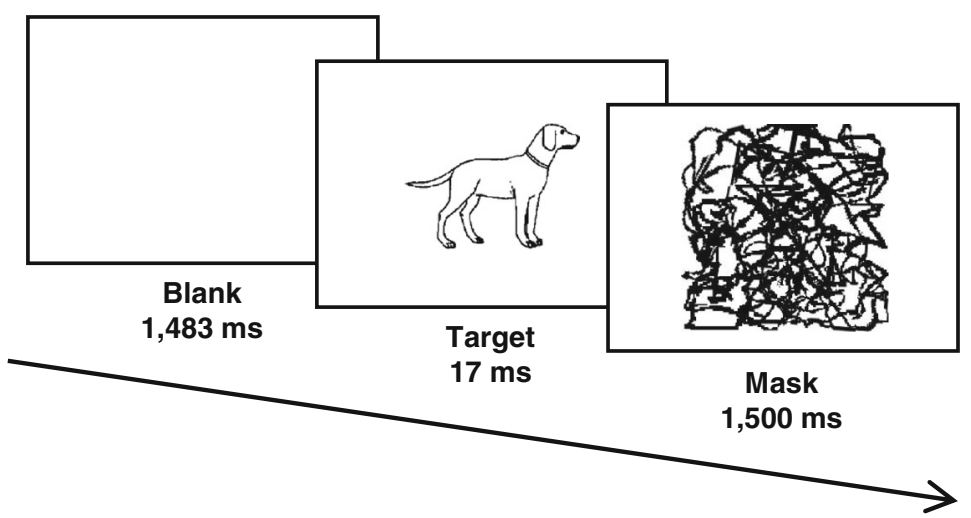

(B) Design based on signal detection theory

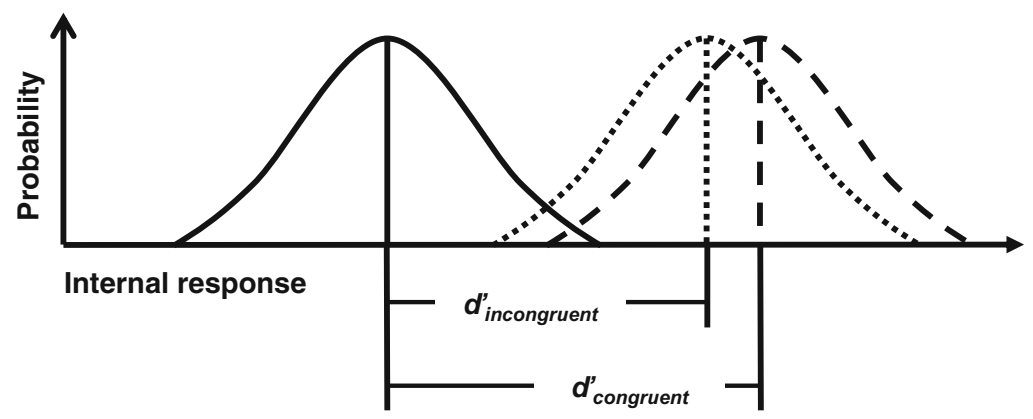

Fig. 1 a Sequence of three frames presented in each trial: A blank, a target picture (e.g., a dog), and a pattern mask. The target picture and pattern mask were presented in black in the center of a white background. b Represents the current experimental design in terms of signal detection theory. The distributions of dashed, dotted, and solid lines represent target present/congruent, target present/incongruent, and target absent conditions, respectively. In this design, the congruent and incongruent conditions share the same FA rate. The sensitivity $\left(d^{\prime}\right)$ was calculated using the equations: $d^{\prime}=z$ (hit rate) $-z$ (FA rate) in the congruent and incongruent conditions, separately (Green \& Swets, 1966; Macmillan \& Creelman, 2005) 
was estimated on the basis of 96 trials (48 pictureabsent trials $\times 2$ blocks; see Table 1); $d^{\prime}$ values were calculated based on the hit and FA rate (see Figs. 1b-2), and then submitted to a three-way analysis of variance (ANOVA) with the factors of congruency, prime type, and SOA (see Table 2 for results). Critically, there was a significant three-way interaction. Paired $t$ tests (Holm-Bonferroni correction, one-tailed were used because higher $d^{\prime}$ in the congruent than the incongruent condition was expected) demonstrated the congruency effect by naturalistic sounds at the 350ms SOA, $t(19)=2.81, p<.05$, but not at the $1,000-\mathrm{ms}$ SOA, $t(19)=-1.63, p=.12$; in contrast, the congruency effect by spoken words occurred at the $1,000-\mathrm{ms}$ SOA, $t(19)=2.87, p<.05$, but not at the $350-\mathrm{ms}$ SOA, $t(19)=0.32, p=.75$. We therefore replicated the results at the $350-\mathrm{ms}$ SOA reported in Chen and Spence (2011).

\section{Experiment 2}

\section{Method}

Thirty-six volunteers (seven males, mean age 19.7 years) took part in this experiment. Three factors were tested with all participants: prime type (naturalistic sound or spoken word), congruency (congruent or incongruent), and SOA ( 350 or $1,000 \mathrm{~ms}$ ). The fourth factor, the order in which the SOAs were tested, was manipulated between participants: Half of the participants were tested with the 350-ms SOA in the first session and the 1,000-ms SOA in the second session (Group 1: 350-1,000 ms); the order was reversed for the remainder of the participants (Group 2: 1,000-350 $\mathrm{ms})$. The stimuli and task were the same as in Experiment 1. The experiment took an hour to complete.

\section{Results}

The participant's $d$ ' (see Fig. 3) was calculated based on the hit and FA rates in each condition (see Table 3), and then

Table 1 Percentage of hit and false alarm (FA) rates ( $S E$ in parentheses) in each of the conditions in Experiment 1

\begin{tabular}{lllll}
\hline \multirow{2}{*}{ SOA (ms) } & Sound type & Hit rate & \multirow{2}{*}{ FA rate } \\
\cline { 3 - 4 } & & Congruent & Incongruent & \\
\hline 350 & Naturalistic sound & $83.4(3.8)$ & $79.1(4.0)$ & $7.1(2.6)$ \\
& Spoken word & $84.0(3.6)$ & $81.9(4.6)$ & $6.7(2.1)$ \\
\multirow{2}{*}{1,000} & Naturalistic sound & $65.3(3.7)$ & $68.3(4.2)$ & $10.3(4.0)$ \\
& Spoken word & $73.8(3.5)$ & $67.3(3.9)$ & $12.4(3.6)$ \\
\hline
\end{tabular}

submitted to a four-way ANOVA (see Table 4a for the results). There was a significant three-way interaction between congruency, prime type, and order. Two separate two-way ANOVAs for each prime type with the factors of congruency and order demonstrated that the congruency effect was significant for naturalistic sounds, $F(1,34)=$ $7.50, p<.05, \eta_{\mathrm{p}}{ }^{2}=0.18$, without being modulated by order (Congruency $\times$ Order: $F<1, p=.87, \eta_{\mathrm{p}}{ }^{2}=0.001$ ). However, for spoken words, the congruency effect was modulated by order (Congruency $\times$ Order): $F(1,34)=$ 12.41, $p<.005, \eta_{\mathrm{p}}{ }^{2}=0.27$. Post hoc tests demonstrated that the congruency effect by spoken words was significant in Group $2(1,000-350 \mathrm{~ms}), t(17)=6.18, p<.001$, but not in Group $1(350-1,000 \mathrm{~ms}), t(17)=0.65, p=.53$. These results therefore suggest that the SOA order influenced the cross-modal semantic congruency effect elicited by spoken words but not by naturalistic sounds. Such a carryover effect of the SOA from one session to the next may mask the modulation of SOA on the congruency effect from auditory cues. The data from the two sessions were therefore analyzed separately.

When only including the data from the first session (top row in Fig. 3), a three-way ANOVA with the factors of congruency, prime type, and SOA (with SOA as a between-participant factor) was conducted (see Table 4b). This is the same design as in Experiment 1, and the results were replicated: The three-way interaction was significant. Paired $t$ tests demonstrated that the congruency effect by naturalistic sounds was only observed at the $350-\mathrm{ms} \mathrm{SOA}$, $t(17)=2.50, p<.05$, but not at the $1,000-\mathrm{ms} \mathrm{SOA}, t(17)=-$ $0.27, p=.79$. In contrast, the congruency effect by spoken words was only statistically significant at the $1,000-\mathrm{ms}$ SOA, $t(17)=3.94, p<.005$, but not at the $350-\mathrm{ms} \mathrm{SOA}$, $t(17)=0.50, p=.62$.

The results of the second session (bottom row in Fig. 3) were different from the first session (see Table 4c). The significant interaction between congruency and SOA was attributed to the fact that the congruency effect was only significant at the $350-\mathrm{ms}$ SOA, $t(17)=4.69, p<.001$, but not at the $1,000-\mathrm{ms}$ SOA, $t(17)=0.16, p=.87$. Planned comparisons demonstrated that the congruency effect was significant at the $350-\mathrm{ms}$ SOA for both naturalistic sounds, $t(17)=2.98$, $p<.05$, and spoken words, $t(17)=4.30, p<.001$, but for neither at the 1,000-ms SOA, $t(17)=-0.57, p=.58$, and $t(17)=0.65, p=.53$, respectively. The significant interaction between congruency and prime type reflected the congruency effect being significant for spoken words, $t(35)=3.48, p<.005$, but only marginally significant for naturalistic sounds, $t(35)=1.88, p=.07$. The latter perhaps results from the slightly higher $d^{\prime}$ in the incongruent than in the congruent condition at the 1,000-ms SOA. 


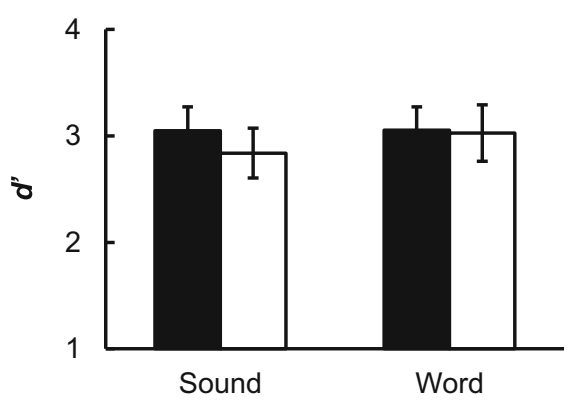

$350 \mathrm{~ms}$

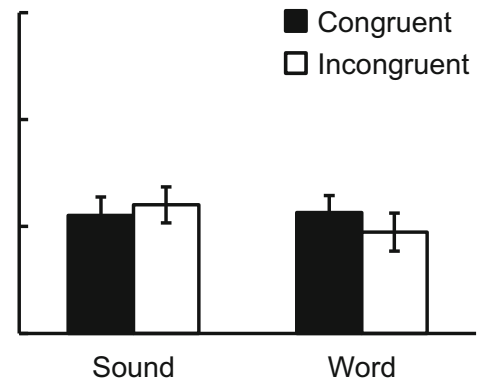

$1000 \mathrm{~ms}$

Fig. 2 Mean sensitivity $\left(d^{\prime}\right)$ at the 350- and 1,000-ms SOAs in Experiment 1. Error bars indicate $\pm 1 S E M$. Sound = naturalistic sounds; Word = spoken words

\section{General discussion}

The results of the present study demonstrate that the presentation of naturalistic sounds enhanced the visual sensitivity of semantically congruent pictures at the shorter SOA $(350 \mathrm{~ms})$ than spoken words did $(1,000 \mathrm{~ms})$ when each participant just encountered either one of the SOAs. The cross-modal semantic priming effects elicited by the presentation of naturalistic sounds versus spoken words can therefore be dissociated in terms of their differing time courses. Furthermore, naturalistic sounds consistently primed the visual pictures at the short SOA; in contrast, the priming effect elicited by spoken words was significantly modulated by the SOA tested beforehand. Specifically, when the 1,000-ms SOA (demonstrating a significant priming effect) was tested first, the priming effect carried over to the 350-ms SOA (this was not observed in Experiment 1). However, when the 350-ms SOA (where no priming effect was observed) was tested first, the priming effect at the 1,000-ms SOA was eliminated as well. Finally, higher sensitivity in the 350-ms than in the 1,000 -ms SOA was observed in both experiments. This can be explained by an attentional cuing effect elicited by the presentation of a temporally close auditory cue (McDonald, TederSälejärvi, \& Hillyard, 2000).

That naturalistic sounds elicited the cross-modal semantic priming effect faster (i.e., at the shorter SOA) than spoken words did suggests that the time required to access meaning for the former is shorter (Chen \& Spence, 2013; Cummings et al., 2006; Saygin et al., 2005). Consistent evidence comes from the results of ERPs studies: For instance, Murray, Camen, Andino, Bovet, and Clarke (2006) have demonstrated that the brain activities associated with naturalistic sounds produced by living versus nonliving things can be discriminated around $70 \mathrm{~ms}$ to $119 \mathrm{~ms}$ after sound onset. The component associated with the meaning of spoken words (the N400; Kutas \& Hillyard, 1980), on the other hand, typically starts $200 \mathrm{~ms}$ after word onset, and it could be delayed if the word is longer or else shares initial syllables with other words (van Petten, Coulson, Rubin, Plante, \& Parks, 1999).

These results may partly be attributed to the nature of the acoustic signals that are associated with each type of stimulus: Naturalistic sounds associated with different object categories have distinct time-frequency spectrums from each other (e.g., Murray et al., 2006). By contrast, spoken words become comprehensible when the acoustic signals are abstracted into various phonetic representations, and the latter are then used to access their associated lexical representations (Obleser \& Eisner, 2009). Consequently, a semantic network suggests that naturalistic sounds and visual pictures access semantics directly, whereas spoken words access their meanings via lexical representations (Chen \& Spence, 2011, 2017; Glaser \& Glaser, 1989). Hence, the cross-modal semantic interactions between naturalistic sounds and pictures would be expected to occur more rapidly than between spoken words and pictures, as demonstrated in the present study.

Table 2 Results of analysis of sensitivity $\left(d^{\prime}\right)$ in Experiment 1 (three-way ANOVA: Congruency $\times$ Prime Type $\times$ SOA)

\begin{tabular}{lllll}
\hline Effect & $F(1,38)$ & $p$ & $\eta_{\mathrm{p}}{ }^{2}$ & Note \\
\hline Congruency & 5.92 & $<.05$ & 0.14 & Congruent $(2.58)>$ Incongruent $(2.50)$ \\
SOA & 11.22 & $<.005$ & 0.23 & $350 \mathrm{~ms}(2.99)>1,000 \mathrm{~ms}(2.10)$ \\
Congruency $\times$ Prime Type $\times$ SOA & 9.99 & $<.005$ & 0.21 & \\
\hline
\end{tabular}




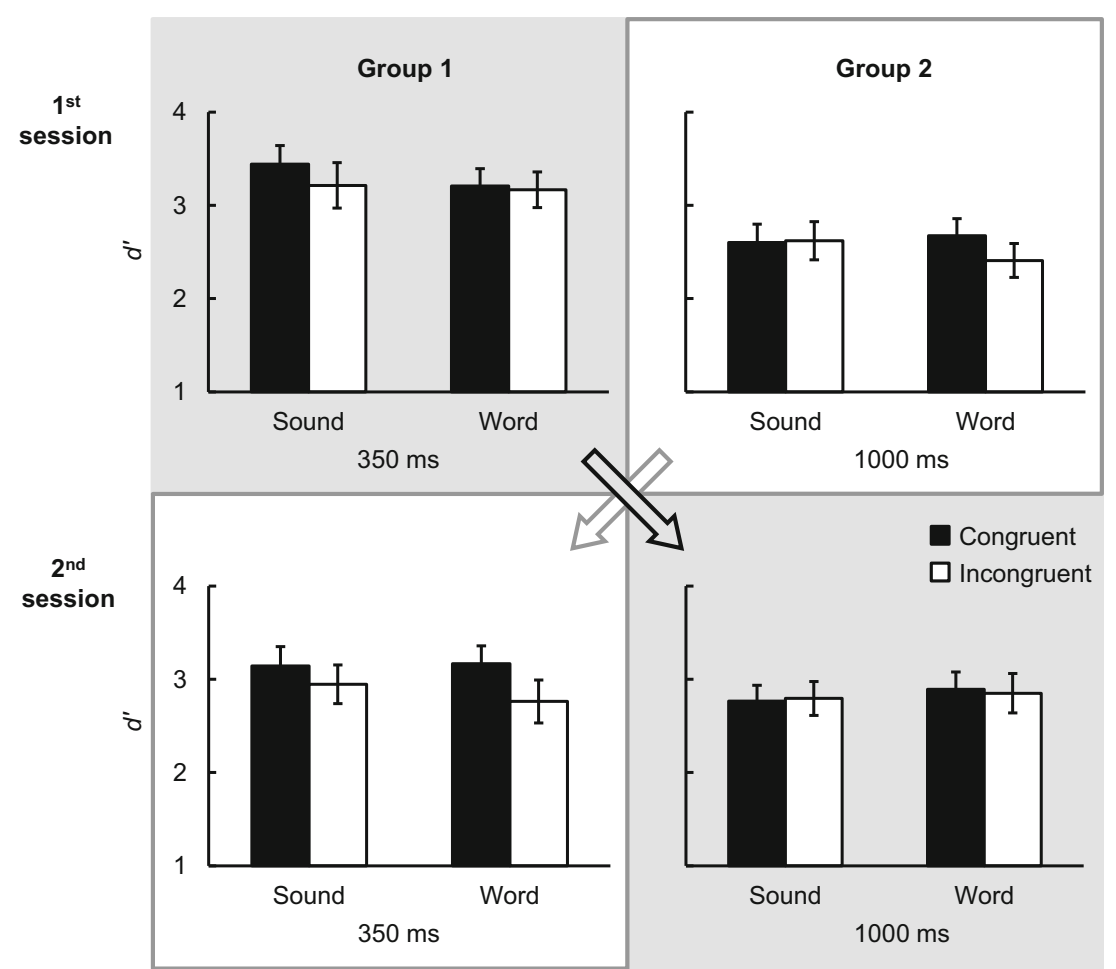

Fig. 3 Mean sensitivity $\left(d^{\prime}\right)$ at the 350- and 1,000-ms SOAs for Group 1 (tested in the order 350- and then 1,000-ms SOA) and Group 2 (with the order reversed) in Experiment 2. Error bars indicate $\pm 1 S E M$. Sound = naturalistic sounds; Word = spoken words

At the 1,000-ms SOA, only spoken words but not naturalistic sounds gave rise to cross-modal semantic priming effects, thus suggesting that the effect induced by naturalistic sounds is short-lived (see also Chen \& Spence, 2017; Kim, Porter, \& Goolkasian, 2014, when using the picture categorization task). Given that naturalistic sounds can access their meaning rapidly (within $350 \mathrm{~ms}$ in the current study), the short-lived priming effect suggests that the activated meaning would be forgotten rapidly as well, unless the information can be temporally maintained. The maintenance of representations of naturalistic sounds, nevertheless, is underpinned by the auditory imagery capability, or else by being transferred into lexical codes and stored in the phonological loop (Snyder \& Gregg, 2011; Soemer \& Saito, 2015), and both processes take extra time or cognitive resources. In contrast, spoken words essentially have the benefit of being maintained in the phonological loop in the working memory system (Baddeley, 2012), thus leading to the significant priming effect over a greater range of SOAs than naturalistic sounds (current study; Chen \& Spence, 2017).

The final contrast lies in the fact that the time course of the cross-modal semantic priming effect by naturalistic sounds was stable, whereas that elicited by spoken words was modulated by the prior context (i.e., the order in which the SOAs were tested). Audiovisual

Table 3 Percentage of hit and false alarm (FA) rates ( $S E$ in parentheses) in each of the conditions in Experiment 2

\begin{tabular}{|c|c|c|c|c|c|}
\hline & \multirow[t]{2}{*}{ SOA (ms) } & \multirow[t]{2}{*}{ Sound type } & \multicolumn{2}{|l|}{ Hit rate } & \multirow[t]{2}{*}{ FA rate } \\
\hline & & & Congruent & Incongruent & \\
\hline \multirow[t]{4}{*}{ Group 1} & \multirow[t]{2}{*}{350 (first session) } & Naturalistic sound & $88.0(2.3)$ & $81.0(4.3)$ & $3.2(1.4)$ \\
\hline & & Spoken word & $82.6(3.8)$ & $81.4(4.0)$ & $2.2(0.6)$ \\
\hline & \multirow[t]{2}{*}{1,000 (second session) } & Naturalistic sound & $71.9(3.7)$ & $72.3(4.1)$ & $2.5(0.9)$ \\
\hline & & Spoken word & $71.4(4.4)$ & $69.0(5.2)$ & $1.4(0.4)$ \\
\hline \multirow[t]{4}{*}{ Group 2} & \multirow[t]{2}{*}{350 (second session) } & Naturalistic sound & $80.7(4.0)$ & $75.8(5.1)$ & $5.6(3.3)$ \\
\hline & & Spoken word & $79.2(3.8)$ & $67.4(5.4)$ & $3.5(2.2)$ \\
\hline & \multirow[t]{2}{*}{ 1,000 (first session) } & Naturalistic sound & $69.4(4.0)$ & $69.7(4.5)$ & $4.7(2.2)$ \\
\hline & & Spoken word & $71.1(4.9)$ & $64.0(5.9)$ & $5.5(2.3)$ \\
\hline
\end{tabular}


Table 4 Results of analysis of sensitivity $\left(d^{\prime}\right)$ in Experiment 2

\begin{tabular}{|c|c|c|c|c|}
\hline Effect & $F(1,34)$ & $p$ & $\eta_{\mathrm{p}}^{2}$ & Note \\
\hline \multicolumn{5}{|c|}{ (A) Four-way ANOVA $($ Congruency $\times$ Prime Type $\times$ SOA $\times$ Order $)$} \\
\hline Congruency & 17.85 & $<.001$ & 0.34 & Congruent (2.99) > Incongruent (2.85) \\
\hline SOA & 45.32 & $<.001$ & 0.57 & $350 \mathrm{~ms}(3.13)>1,000 \mathrm{~ms}(2.70)$ \\
\hline Congruency $\times \mathrm{SOA}$ & 10.15 & $<.005$ & 0.23 & \\
\hline Congruency $\times$ Prime Type & 6.46 & $<.05$ & 0.16 & \\
\hline Congruency $\times$ Order & 4.49 & $<.05$ & 0.12 & \\
\hline Congruency $\times$ Prime Type $\times$ Order & 16.95 & $<.001$ & 0.33 & \\
\hline \multicolumn{5}{|c|}{ (B) First session: three-way ANOVA (Congruency $\times$ Prime Type $\times$ SOA) } \\
\hline Congruency & 8.85 & $<.01$ & 0.21 & Congruent (2.98) > Incongruent (2.85) \\
\hline SOA & 6.65 & $<.05$ & 0.16 & $350 \mathrm{~ms}(3.26)>1,000 \mathrm{~ms}(2.58)$ \\
\hline Congruency $\times$ Prime Type $\times$ SOA & 12.24 & $<.005$ & 0.27 & \\
\hline \multicolumn{5}{|c|}{ (C) Second session: three-way ANOVA (Congruency $\times$ Prime Type $\times$ SOA) } \\
\hline Congruency & 15.83 & $<.001$ & 0.32 & Congruent (2.99) > Incongruent (2.84) \\
\hline Congruency $\times \mathrm{SOA}$ & 14.41 & $<.005$ & 0.30 & \\
\hline Congruency $\times$ Prime Type & 4.96 & $<.05$ & 0.13 & \\
\hline
\end{tabular}

integration/interactions involving speech sounds have been demonstrated to be flexible. For example, the cross-modal semantic priming effect by spoken words can be speeded up so as to be observed at around $350-\mathrm{ms} \mathrm{SOA}$ if the participants have been exposed to the longer SOA condition (the current study) or if the participants have to identify the target picture by reporting its name (Chen \& Spence, 2011). In addition, the integration of verbal cues and visual lip movements occurs more often (indexed by a larger McGurk effect; McGurk \& McDonald, 1976) if a series of congruent (compared to incongruent) audiovisual speech stimuli were presented beforehand (Nahorna, Berthommier, \& Schwartz, 2012). Finally, the digits and letters that are presented subliminally to both vision and audition would be integrated only if the participants had consciously experienced these pairings prior to the test (Faivre, Mudrik, Schwartz, \& Koch, 2014). The higher flexibility of audiovisual interactions involving spoken words than naturalistic sounds at the semantic level perhaps stems from the former accessing the semantic representations at an abstract, categorical, and modality-insensitive level, whereas the latter served as modality-specific and contextdependent attributes associated with the object crossmodally (Edmiston \& Lupyan, 2015; Waxman \& Gelman, 2009).

Together, the results of the two experiments reported here demonstrate that naturalistic sounds elicit more rapid cross-modal priming than do spoken words, which is likely determined by their speed of semantic access stemming from the different processing routes. On the other hand, the advantage of spoken words over naturalistic sounds to prime visual pictures across a more prolonged prime-target interval should result from the former being better maintained in working memory. Finally, consistent with previous studies, interactions between spoken words and visual signals are flexible - that is, they can be enhanced or inhibited by prior context or by task demands.

Acknowledgements The authors are supported by the Arts and Humanities Research Council (AHRC), Rethinking the Senses grant (AH/L007053/1).

\section{Appendix}

The auditory stimuli used in the present study. Note that the lengths of the naturalistic sound and spoken word referring to the same picture were matched at $350 \mathrm{~ms}$ for the 14 one-syllable words, $450 \mathrm{~ms}$ for the seven two-syllable words, and $500 \mathrm{~ms}$ for the three(or more) syllable words (three words). The identification accuracy, confidence, and familiarity ratings (maximum score $=7$ ) for the sounds reflect the mean performance of 18 participants (four males, mean age 28 years, reported in Chen \& Spence, 2011). The ratings of imagery concordance (maximum score $=5$ ) were acquired via an online study (30 participants for naturalistic sounds, 18 males, mean age 31 years; 30 participants for spoken words, 19 males, mean age 33 years). All scores were lower for naturalistic sounds than for spoken words. Identification accuracy: $t(23)=5.42$, $p<.001$; confidence rating: $t(25)=8.33, p<.001$; familiarity rating: $t(26)=8.38, p<.001$; imagery concordance: $t(24)=4.24, p<.001 ;$ two-tailed, unequal variance assumed 


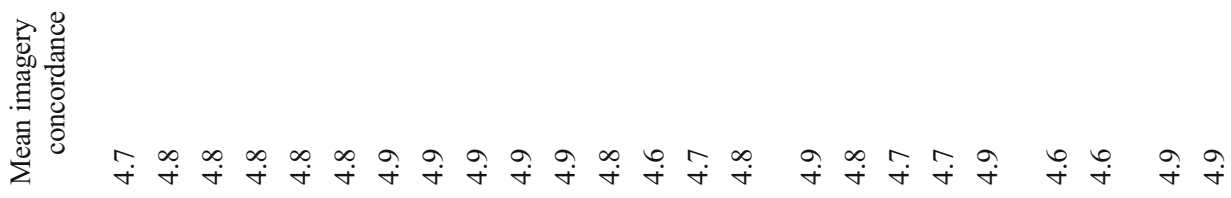

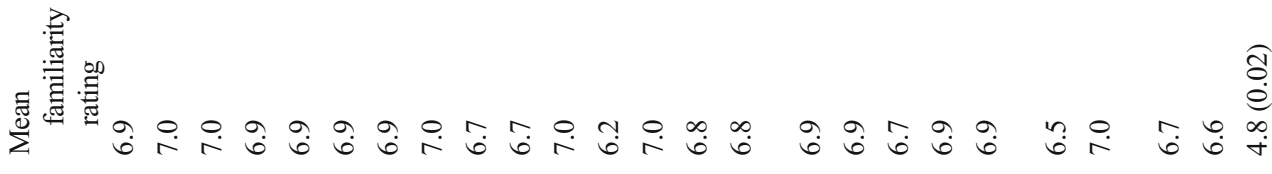

焉

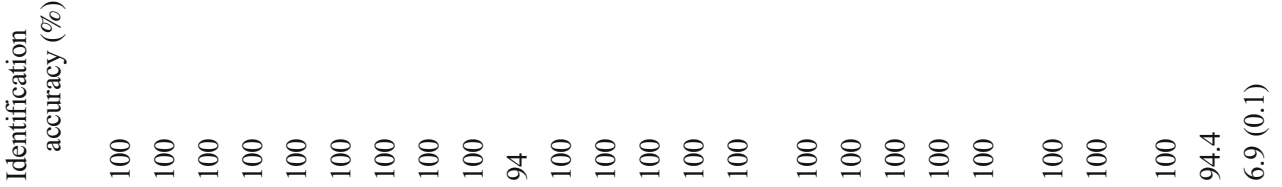

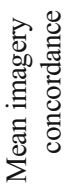

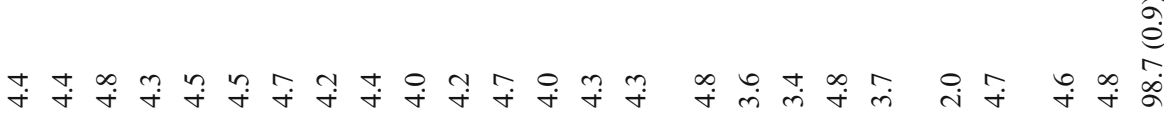

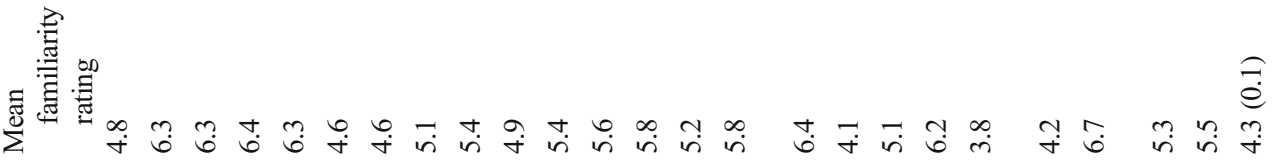

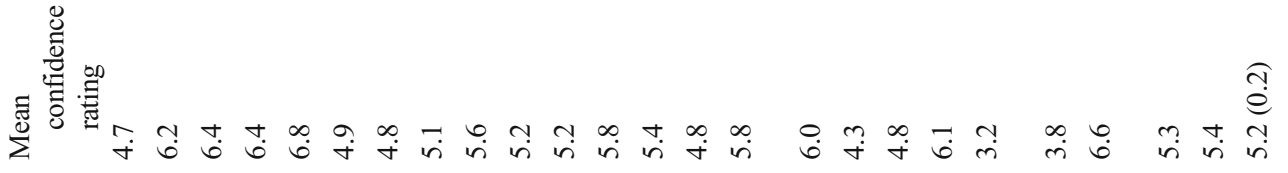

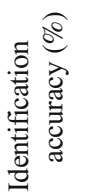

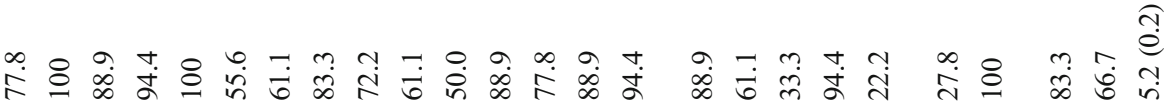

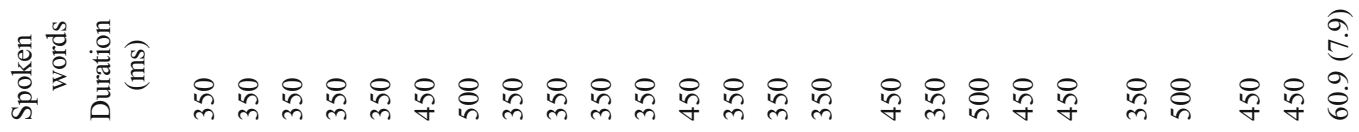

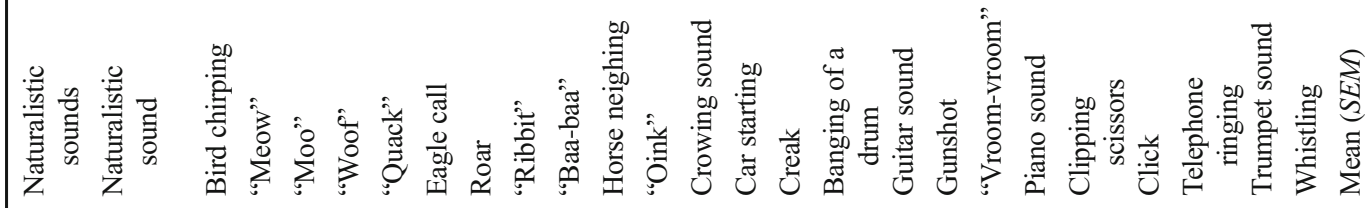

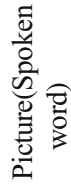

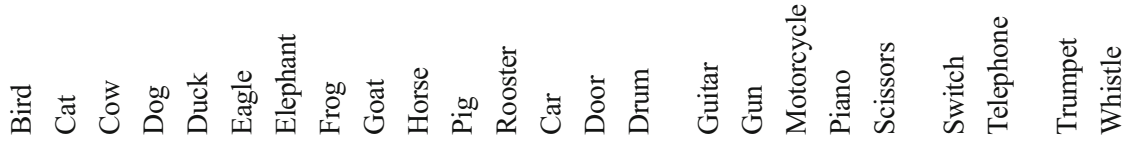


The stimuli used in the practice sessions were fly (humming fly), tiger (tiger roaring), bell (bell ringing), and cannon (cannon fire)

Open Access This article is distributed under the terms of the Creative Commons Attribution 4.0 International License (http:// creativecommons.org/licenses/by/4.0/), which permits unrestricted use, distribution, and reproduction in any medium, provided you give appropriate credit to the original author(s) and the source, provide a link to the Creative Commons license, and indicate if changes were made.

\section{References}

Baddeley, A. (2012). Working memory: Theories, models, and controversies. Annual Review of Psychology, 63, 1-29.

Barsalou, L. W., Santos, A., Simmons, W. K., \& Wilson, C. D. (2008). Language and simulation in conceptual processing. In M. de Vega, A. Glenberg, \& A. Graesser (Eds.), Symbols and embodiment: Debates on meaning and cognition (pp. 245-283). Oxford: Oxford University Press.

Bates, E., D’Amico, S., Jacobsen, T., Székely, A., Andonova, E., Devescovi, A., \& Tzeng, O. (2003). Timed picture naming in seven languages. Psychonomic Bulletin \& Review, 10, 344-380.

Boutonnet, B., \& Lupyan, G. (2015). Words jump-start vision: A label advantage in object recognition. Journal of Neuroscience, 35, 9329-9335.

Chen, Y.-C., \& Spence, C. (2010). When hearing the bark helps to identify the dog: Semantically-congruent sounds modulate the identification of masked pictures. Cognition, 114, 389-404.

Chen, Y.-C., \& Spence, C. (2011). Crossmodal semantic priming by naturalistic sounds and spoken words enhances visual sensitivity. Journal of Experimental Psychology: Human Perception and Performance, 37, 1554-1568.

Chen, Y.-C., \& Spence, C. (2013). The time-course of the cross-modal semantic modulation of visual picture processing by naturalistic sounds and spoken words. Multisensory Research, 26, 371-386.

Chen, Y.-C., \& Spence, C. (2017). Comparing audiovisual semantic interactions between linguistic and non-linguistic stimuli. Manuscript submitted for publication.

Cummings, A., Čeponienè, R., Koyama, A., Saygin, A. P., Townsend, J., $\&$ Dick, F. (2006). Auditory semantic networks for words and natural sounds. Brain Research, 1115, 92-107.

Edmiston, P., \& Lupyan, G. (2015). What makes words special? Words as unmotivated cues. Cognition, 143, 93-100.

Faivre, N., Mudrik, L., Schwartz, N., \& Koch, C. (2014). Multisensory integration in complete unawareness: Evidence from audiovisual congruency priming. Psychological Science, 25, 2006-2016.

Glaser, W. R., \& Glaser, M. O. (1989). Context effects in Stroop-like word and picture processing. Journal of Experimental Psychology: General, 118, 13-42.
Green, D. M., \& Swets, J. A. (1966). Signal detection theory and psychophysics. New York: Wiley.

Kim, Y., Porter, A. M., \& Goolkasian, P. (2014). Conceptual priming with pictures and environmental sounds. Acta Psychologica, 146, 73-83.

Kutas, M., \& Hillyard, S. A. (1980). Reading senseless sentences: Brain potentials reflect semantic incongruity. Science, 207, 203-205.

Lupyan, G., \& Thompson-Schill, S. L. (2012). The evocative power of words: Activation of concepts by verbal and nonverbal means. Journal of Experimental Psychology: General, 141, 170-186.

Lupyan, G., \& Ward, E. J. (2013). Language can boost otherwise unseen objects into visual awareness. Proceedings of the National Academy of Sciences of the United States of America, 110, 14196-14201.

Macmillan, N. A., \& Creelman, C. D. (2005). Detection theory: A user's guide. Mahwah, NJ: Erlbaum.

Magnié, M. N., Besson, M., Poncet, M., \& Dolisi, C. (2003). The Snodgrass and Vanderwart set revisited: Norms for object manipulability and for pictorial ambiguity of objects, chimeric objects, and nonobjects. Journal of Clinical and Experimental Neuropsychology, $25,521-560$

McDonald, J. J., Teder-Sälejärvi, W. A., \& Hillyard, S. A. (2000). Involuntary orienting to sound improves visual perception. Nature, 407, 906-908.

McGurk, H., \& MacDonald, J. (1976). Hearing lips and seeing voices. Nature, 264, 746-748.

Murray, M. M., Camen, C., Andino, S. L. G., Bovet, P., \& Clarke, S. (2006). Rapid brain discrimination of sounds of objects. Journal of Neuroscience, 26, 1293-1302.

Nahorna, O., Berthommier, F., \& Schwartz, J. L. (2012). Binding and unbinding the auditory and visual streams in the McGurk effect. Journal of the Acoustical Society of America, 132, 1061-1077.

Obleser, J., \& Eisner, F. (2009). Pre-lexical abstraction of speech in the auditory cortex. Trends in Cognitive Sciences, 13, 14-19.

Saygin, A. P., Dick, F., \& Bates, E. (2005). An on-line task for contrasting auditory processing in the verbal and nonverbal domains and norms for younger and older adults. Behavior Research Methods, 37, 99-110.

Snodgrass, J. G., \& Vanderwart, M. (1980). A standardized set of 260 pictures: Norms for name agreement, image agreement, familiarity, and visual complexity. Journal of Experimental Psychology: Human Learning and Memory, 6, 174-215.

Snyder, J. S., \& Gregg, M. K. (2011). Memory for sound, with an ear toward hearing in complex auditory scenes. Attention, Perception, \& Psychophysics, 73, 1993-2007.

Soemer, A., \& Saito, S. (2015). Maintenance of auditory-nonverbal information in working memory. Psychonomic Bulletin \& Review, 22, 1777-1783.

Van Petten, C., Coulson, S., Rubin, S., Plante, E., \& Parks, M. (1999). Time course of word identification and semantic integration in spoken language. Journal of Experimental Psychology: Learning, Memory, and Cognition, 25, 394-417.

Waxman, S. R., \& Gelman, S. A. (2009). Early word-learning entails reference, not merely associations. Trends in Cognitive Sciences, 13, 258-263. 